Check for updates

Cite this: RSC Adv., 2019, 9, 26214

Received 9th April 2019

Accepted 5th August 2019

DOI: $10.1039 / c 9 r a 02644 d$

rsc.li/rsc-advances

\section{Structural factors of benzylated glucopyranans for shear-induced adhesion $\uparrow$}

\author{
Abu Bin Insan, (D) ${ }^{a}$ Yuta Kawaguchi, ${ }^{\text {b }}$ Hiromichi Inokuchi, ${ }^{\text {b }}$ Hiroshi Endo ${ }^{\text {b }}$ \\ and Yasuhito Koyama (D) *a
}

We have prepared benzylated glucopyranans and evaluated the structural effects on the adhesion capacity. It was found that $97 \%$-benzylated $(1 \rightarrow 2)$-glucopyranan exhibited a unique shear-induced adhesion. The effects of structural factors on the adhesion behaviors are discussed through systematic adhesion tests, differential scanning calorimetry, theoretical models, and IR spectroscopy.
The development of new bioglues has been strongly urged from the viewpoint of advancing biomedical engineering. ${ }^{1-4}$ The shear-induced adhesion behaviors of von Willebrand factor $(\mathrm{VWF})^{5-7}$ is a nice motif to create a new bioglue. VWF is a mosaic plasma protein. At the sites of vascular injury, shear and elongated flows of fluid alter the original coiled structure of VWF to an elongated structure. The shear-induced structural change of VWF exposes the multiple binding sites of VWF to receptors on platelets and collagen, which remarkably increases the adhesion of VWF, resulting in hemostasis or thrombosis. It is noted that the original coiled structure of VWF hardly exhibits adhesive properties. Apart from the adhesion mechanism of VWF, such shear-induced adhesion analogous to that of VWF should be actively studied as the function of bioglues, which could provide unprecedented bioglues with mechanical responsivity, stress-resistance, and readhesion capacity in the future. However, the structure of VWF comprising the permutation of 20 natural amino acids is too complicated to generalize the shear-induced adhesion behavior.

Based on this background, we have focused on the simple structures of glucopyranans (Glcps) and their potential applicability to adhesive materials possessing shear-induced adhesion. Glcp is a member of polysaccharides and consists of glucose as a repeating unit, whose structure should be simpler than that of VWF. Gelatinized starch has been long used as an adhesive material for paper products and food additives. ${ }^{8-11}$ Amylose as a Glcp bearing 1,4-glycosidic bonds [Glcp-(1-4)] is the ingredient of starch (Fig. 1a). The adhesion of amylose is densely related to the formation of hydrogen bonds. Conversely,

${ }^{a}$ Department of Pharmaceutical Engineering, Faculty of Engineering, Toyama Prefectural University, 5180 Kurokawa, Imizu, Toyama 939-0398, Japan. E-mail: ykoyama@pu-toyama.ac.jp

${ }^{b}$ Department of Mechanical Systems Engineering, Faculty of Engineering, Toyama Prefectural University, 5180 Kurokawa, Imizu, Toyama 939-0398, Japan

$\dagger$ Electronic supplementary information (ESI) available: Experimental section, ${ }^{1} \mathrm{H}$ NMR, ${ }^{13} \mathrm{C}$ NMR, IR spectra, lap-shear test results for adhesion, and DSC profiles. See DOI: $10.1039 / \mathrm{c} 9 \mathrm{ra} 02644 \mathrm{~d}$ the complete protection of hydroxyl groups in amylose can prevent the adhesion. Thus, we envisioned the preparation of nearly preprotected amylose as a candidate for the shearresponsive adhesive material, strongly assuming the following idea: if the mechanical stress to the polymer exposes the interchain binding sites, such as hydrogen bonding exposed, the polymer should serendipitously show an increase in the adhesive strength $\left(S_{\text {Adh }}\right)$ after applying stress. To assess the postulation, we primarily prepared benzylated Glcp-(1-4) as the glue. Moreover, we have recently reported a new synthetic method of glycosides grafting $(1 \rightarrow 2)$-glucopyranan [Glcp-(1-2)] (Fig. 1b). ${ }^{\mathbf{1 2}}$ Since a series of synthetic Glcp-(1-2) derivatives was available in our group, we also prepared benzylated Glcp-(1-2)-X

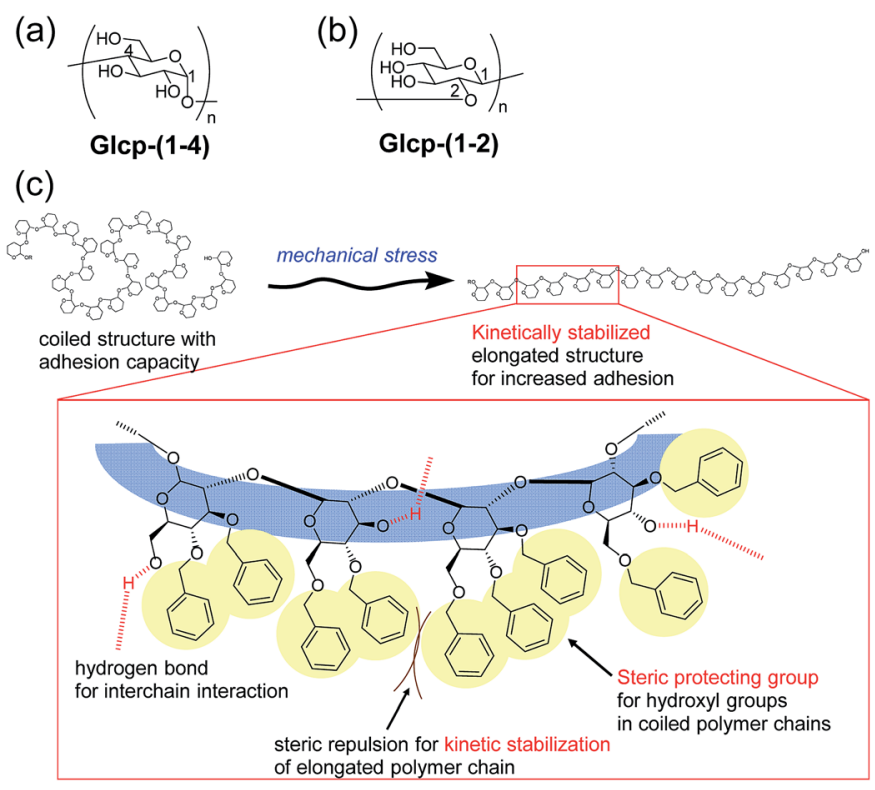

Fig. 1 (a) Structures of Glcp-(1-4) and (b) Glcp-(1-2) and (c) the plausible structure of the kinetically stabilized elongated chain of adhesive Glcp-(1-2)- $X$. 
with different benzylation ratios $(X \%)$ as the additional candidate of the glue.

Herein, we investigated lap-shear tests using two glass plates with Glcp-based glues to evaluate the effects of the structure, stretching velocity $(v)$, shear modulus $(G)$, and water content on $S_{\text {Adh }}$, as well as the shear-induced adhesion behavior. We found that Glcp-(1-2)-97 exhibited a unique shear-induced adhesion. However, the polymer as casted on the glass hardly exhibited adhesive properties, the uniaxial orientation of the polymer by shear stress afforded a remarkable increase in $S_{\mathrm{Adh}}$. It was indicated that the benzyl groups of Glcp-(1-2)-97 appeared to be steric protecting groups not only to hinder the alcohols in the original coiled structure but also to kinetically stabilize the shear-induced elongated polymer structure (Fig. 1c).

Benzylated Glcp-(1-4) was prepared by treating amylose (synthetic, $M_{\mathrm{n}} 31 \mathrm{kDa}$ ) with benzyl chloride and powdered $\mathrm{NaOH}$ in DMSO according to the literature. ${ }^{13}$ By the ${ }^{1} \mathrm{H}$ NMR spectrum, the benzylation ratio was determined to be $95 \% .{ }^{14} \mathrm{We}$ performed differential scanning calorimetry (DSC) measurements on the polymers. The glass transition temperature $\left(T_{\mathrm{g}}\right)$ of Glcp-(1-4)-95 was found to be $23.7^{\circ} \mathrm{C} .{ }^{14}$ With the obtained Glcp(1-4)-95 in hand, we performed lap-shear tests using two glass plates with Glcp-(1-4)-95 as a glue to clarify the adhesion behaviors. The polymer was homogeneously coated on a glass plate with an area of $20 \times 25 \mathrm{~mm}$, and another glass plate was attached to the polymer-coated area (Fig. 2a). Both ends of the glass plates were clamped to a tensile machine. Initially, shear adhesive tests on the polymer samples were performed at a stretching velocity of $10 \mathrm{~mm} \min ^{-1}$. However, even after the repeated application of stress to the glue, Glcp-(1-4)-95 showed negligible adhesion (0.05 $\mathrm{kPa})$, implying that the original structure of Glcp-(1-4)-95 might be rigid and sufficiently stable, which may hardly expose interchain binding sites after applying mechanical stress.

Thus, we prepared Glcp-(1-2)-based adhesives, as shown in Scheme 1 , because the main chain can be regarded as a functionalized polyoxyethylene that seems to be more flexible than that of amylose. We selected 4-penten-1-ol as an initiator, because the terminal olefinic proton signals in the ${ }^{1} \mathrm{H}$ NMR spectrum would be a diagnostic tool to estimate the degree of polymerization (DP) and the number-average molecular weight $\left(M_{\mathrm{n}}\right)$. Sugar-based cyclic sulfite (1) was treated with a catalytic amount of trifluoromethanesulfonic acid (TfOH) in the presence of 4-penten-1-ol ( 2 mol\%) and molecular sieves of $3 \AA$ (MS $3 \mathrm{~A}$ ) in $\mathrm{CH}_{2} \mathrm{Cl}_{2}$ at room temperature. ${ }^{12}$ The required reaction time was $7 \mathrm{~d}$ for the completion of reaction. The structure of the obtained polymer [Glcp-(1-2)-100] was confirmed by IR, ${ }^{1} \mathrm{H}$ NMR, and ${ }^{13} \mathrm{C}$ NMR spectra. ${ }^{\mathbf{1 4}}$ The IR spectrum of Glcp-(1-2)-100 does not include the absorption signal attributed to the sulfite linkage at around $1200 \mathrm{~cm}^{-1},{ }^{\mathbf{1 4}}$ suggesting the perfect elimination of $\mathrm{SO}_{2}$ from the main chain. From the integral ratio between the internal olefin and the aromatic proton signals in the ${ }^{1} \mathrm{H}$ NMR spectrum, ${ }^{14}$ we estimated the DP and $M_{\mathrm{n}}$ to be 51.2 and $22 \mathrm{kDa}$, respectively. The results indicate that the polymerization degree is controllable by the feed ratio of the initiator. The anomeric stereochemistry was almost $\beta(>95 \%)$, which was estimated by the ${ }^{13} \mathrm{C}$ NMR spectrum using the integral ratio (a)

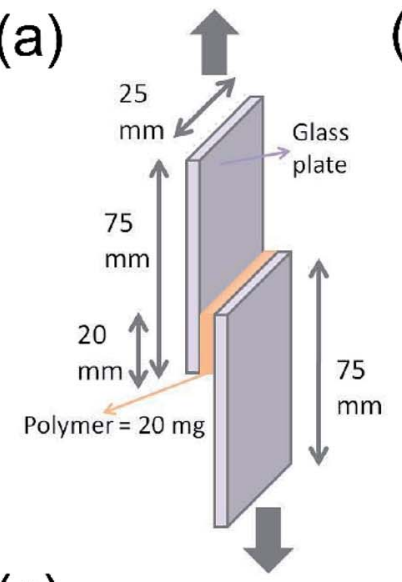

(c)

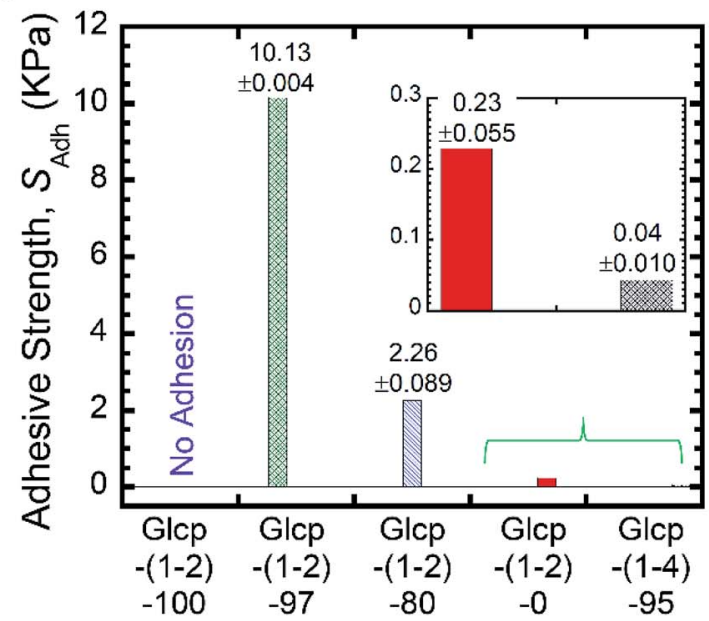

Fig. 2 (a) Adhesion testing geometry to measure $S_{\text {Adh, }}$ (b) a photograph of the macroscopic fiber formation of Glcp-(1-2)-97, and (c) the dependence of $S_{\text {Adh }}$ on the benzylation ratio. Bars are average values from three samples. The relative standard deviations (\%RSD) are $0.04 \%$ for Glcp-(1-2)-97, 3.9\% for Glcp-(1-2)-80, 24\% for Glcp-(1-2)-0, and $23 \%$ for Glcp-(1-4)-95

between the carbon signals at 110-100 ppm for $\beta$ carbons and those at 100-90 ppm for $\alpha$ carbons, ${ }^{\mathbf{1 4}}$ implying that the cationic chain propagation proceeded in an inversion manner via the

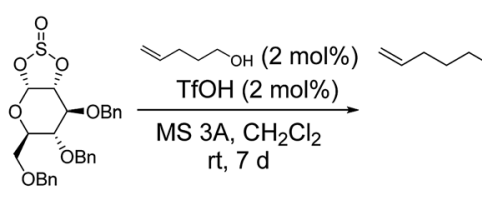

(1)

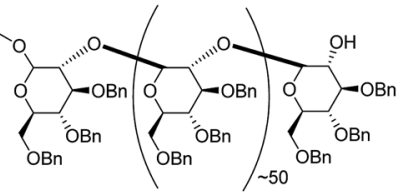

GIcp-(1-2)-100

DP: $51.2, M_{\mathrm{n}} 22 \mathrm{kDa}$ anomeric centers: $\beta(>95 \%)$

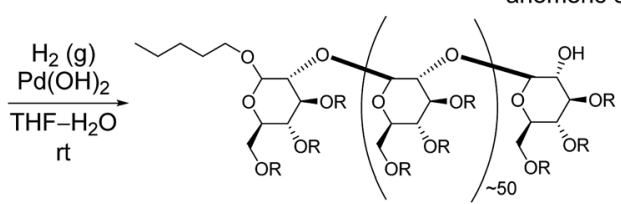

GIcp-(1-2)-X

$\mathrm{R}=\mathrm{Bn}$ or $\mathrm{H}, \mathrm{X}=97,80$, or 0

Scheme 1 Synthesis of Glcp-(1-2)-based adhesives. Glcp-(1-2) with $X \%$ benzylation is abbreviated as Glcp-(1-2)- $X$. 
successive pseudo $\mathrm{S}_{\mathrm{N}} 2$ reactions of alcohol at the anomeric centers. To obtain Glcp-(1-2)- $\boldsymbol{X}$ with different benzylation ratios, we next performed the hydrogenolysis of Glcp-(1-2)-100 using $\mathrm{Pd}(\mathrm{OH})_{2}$ as a catalyst. The mixture was stirred for different reaction intervals under $\mathrm{H}_{2}$ atmosphere to furnish three types of polymers with 97,80 , and $0 \%$ benzylation ratios, where the benzylation ratio was determined by the integral ratio between the aromatic proton signals at $6.8-7.8 \mathrm{ppm}$ and the aliphatic proton signals at 3.0-5.5 ppm in the ${ }^{1} \mathrm{H}$ NMR spectra. The glass transition temperatures $\left(T_{\mathrm{g}}\right)$ were $-5.6{ }^{\circ} \mathrm{C}$ for Glcp-(1-2)-100, 13.7 ${ }^{\circ} \mathrm{C}$ for Glcp-(1-2)-97, $19.3{ }^{\circ} \mathrm{C}$ for Glcp-(1-2)-80, and $29.8{ }^{\circ} \mathrm{C}$ for Glcp-(1-2)-80. ${ }^{\mathbf{1 4}}$ These results suggest that the benzyl protection makes the polymer chain plasticized and benzylated Glcp-(1-2) is more flexible than benzylated Glcp-(1-4).

We performed the lap-shear tests of all Glcp-(1-2) derivatives using three samples to obtain the average values. As a result, it turned out that the order of $S_{\text {Adh }}$ was Glcp-(1-2)-80 $(2.3 \mathrm{kPa})>$ Glcp-(1-2)-97 (0.8 kPa) > Glcp-(1-2)-0 (0.3 kPa), while for Glcp-(12)-100 was not possible to measure $S_{\mathrm{Adh}}$. In order to confirm the readhesion capacity of the polymers, the detached glass plates were joined again by pressing them slightly with a finger for $30 \mathrm{~s}$ at room temperature after the first measurement (Cycle 1), followed immediately by the lap-shear test for subsequent cycles [Cycles 2-6, Fig. 3a (green filled circles) and ESI Fig. S10a $\dagger]^{14}$ Surprisingly, the $S_{\text {Adh }}$ of Glcp-(1-2)-97 increased remarkably from the $1^{\text {st }}$ to $5^{\text {th }}$ cycle and then saturated at around $10 \mathrm{kPa}$, which is stronger than the reported value for fibrin glue $(7 \mathrm{kPa}){ }^{4}$ The results are in a good contrast to that of Glcp-(1-4)-95 with a similar benzylation ratio, clearly suggesting the impact of the glycosidic position on the adhesion capacity of Glcps. After applying stress, Glcp-(1-2)-97 was also found to show a highly viscoelastic nature, which easily formed a fiber (Fig. 2b). Moreover, the $S_{\mathrm{Adh}}$ values of Glcp-(1-2)-80 and Glcp-(1-2)0 hardly changed after the repeated tests. Fig. 2c summarizes the adhesibility results in terms of the saturated $S_{\text {Adh }}$ values after the repeated tests. These results suggest that the $S_{\mathrm{Adh}}$ values increase along with the increase in the benzylation ratio, expect for Glcp-(1-2)-100, seem to be correlated to the $T_{\mathrm{g}}$ values. To know the effects of hydrogen bonds on $S_{\mathrm{Adh}}$, the glass plate coating Glcp-(1-2) was completely dried at $90{ }^{\circ} \mathrm{C}$ under reduced pressure for $24 \mathrm{~h}$ and tested for repeated adhesion capacity. The obtained $S_{\mathrm{Adh}}$ of the dried adhesive was quite high at Cycle 1, which reached approximately $263 \pm 12 \mathrm{kPa}$ but decreased with the repeated tests (Fig. 3a, green open circles). The tendency of the repeated tests appears to be opposite to the wet polymer, indicating not only the reinforcement of adhesion based on the efficient interchain hydrogen bonding but also the role of water as a plasticizer. Moreover, the $S_{\text {Adh }}$ value of Glcp-(1-2)-80 was not related to the repeated cycle.

In order to understand the effects of $v$ on the adhesion, we subsequently investigated the dependency of $S_{\text {Adh }}$ of Glcp-(1-2)97 on $v$ (Fig. $3 \mathrm{~b}$ and ESI Fig. S10b and $\mathrm{c} \dagger) .{ }^{\mathbf{1 4}}$ The adhesion of Glcp-(1-2)-97 between the glass plates was clearly sensitive to $v$. The $S_{\text {Adh }}$ values are plotted as a function of $v$, which is described by a power-law expression. Consequently, it turned out that the $S_{\text {Adh }}$ values were correlated to $v$ to the power of 0.6. According to the theoretical prediction by Schallamach, ${ }^{\mathbf{1 5}}$ the adhesion force between ideal elastomers would correlate to $v$ in a power-law manner with an exponent of $2 / 3$. Thus, the experimental exponent value indicates that the adhesive properties of Glcp-(1-2)-X would almost coincide with those of an elastomer. We also evaluated the correlations between $S_{\text {Adh }}$ and $G$ for all tested samples (Fig. 3c), considering that the Chernyak-Leonov model for the friction of viscoelastic materials anticipates a proportional relationship of $S_{\text {Adh }}$ to $G^{0.5} \cdot{ }^{16-18}$ All plots appear to be straight lines, regardless of different structures and $v$. The $G$ values for all plots are below $10^{5} \mathrm{~Pa}$, which are within the empirical criterion for adhesive materials proposed by Dahlquist. ${ }^{19-21}$ The approximate straight line indicates that $S_{\mathrm{Adh}}$ is correlated to $G$ to the power of 0.55 , which agrees with the theoretical Chernyak-Leonov model. The results obtained from Fig. $3 \mathrm{~b}$ and $\mathrm{c}$ provide evidence for the high viscoelasticity of Glcp-(1-2)-97 as well as the efficient energy dissipation capacity of Glcp-(1-2)-97 on adhesion.

We will now organize the observed adhesive behaviors of Glcp-based adhesives. Based on the low $T_{\mathrm{g}}$ and the mechanical responsivity of Glcp-(1-2)-97 as an ideal elastomer, the coiled polymer structure of Glcp-(1-2)-97 in the virgin adhesive would uniaxially align in the tensile direction to give the elongated structure during the lap-shear tests as expected in Fig. 1c. The rigid and bulky repeating structure of Glcp-(1-2)-97 may kinetically suppress the structural relaxation due to the steric repulsion. In the cases of Glcp-(1-2)-80 and Glcp-(1-2)-0, the increased $T_{\mathrm{g}} \mathrm{S}$ would make the mechanical responsivity weaken (a)

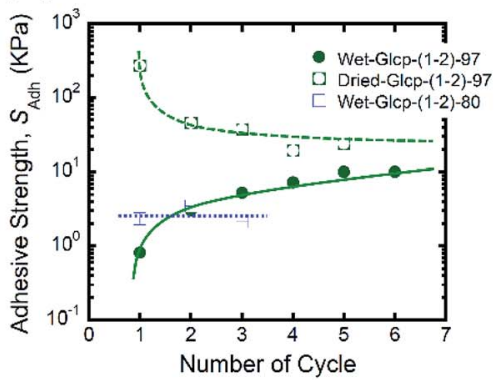

(b)

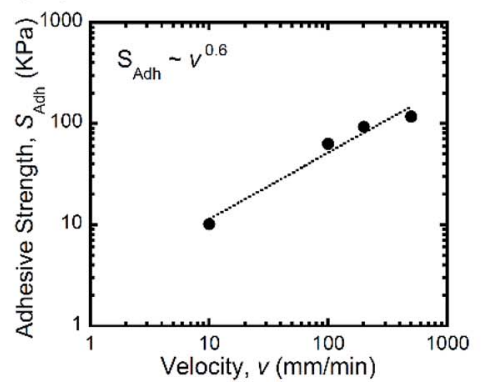

(c)

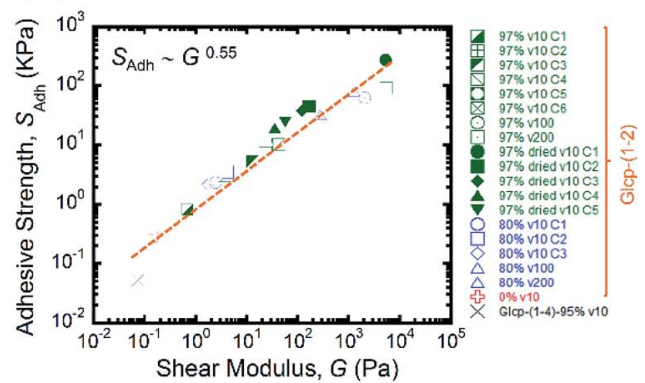

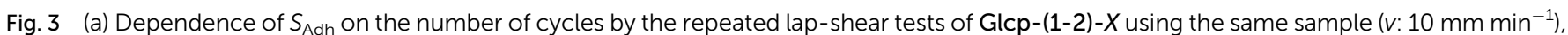
(b) dependence of the $S_{\text {Adh }}$ value of Glcp-(1-2)-97 on $v$, and (c) the proportional relationship of $S_{\text {Adh }}$ to $G^{0.55}$. Lines are to guide the eyes. 
and these less bulky repeating units would hardly prevent the reformation of the original coiled structure. It is highlighted that the elongated structure of Glcp-(1-2)-97 shows a remarkably high $S_{\text {Adh }}$ than the virgin polymer as a coiled structure. The shear-induced adhesive change in Glcp-(1-2)-97 seems to be similar to that of VWF in blood as described in the introduction. In a similar manner to VWF, the elongated polymer chain of Glcp-(1-2)-97 is expected to expose the hydrogen bonds as intermolecular binding sites, leading to an increase in $S_{\mathrm{Adh}}$. It is interesting that Glcp-(1-2)-97 exhibits the strongest $S_{\mathrm{Adh}}$ after applying stress in spite of the least content of hydroxyl groups in the polymers. The results imply that after applying stress the hydroxyl groups in Glcp-(1-2)-97 would facilitate the interchain hydrogen bond formations as physical cross-links. To evaluate the special hydrogen bonding capacity in Glcp-(1-2)-97, we compared the IR spectra of the polymers (Fig. 4). In the spectra of both Glcp-(1-2)-80 and Glcp-(1-4)-95, the absorbance signals of the hydroxyl groups appear at around $3300 \mathrm{~cm}^{-1}$, which indicate the strong hydrogen bonding of the hydroxyl groups in these polymers. ${ }^{22}$ Moreover, the signal of virgin Glcp-(1-2)-97 appears at $3427 \mathrm{~cm}^{-1}$, which could be assigned to free $\mathrm{OH}^{22}$ This means that the hydroxyl groups in virgin Glcp-(1-2)-97 would hardly contribute to the intra- and intermolecular interactions. The exposed hydroxyl groups after applying stress would facilitate interchain hydrogen bonding, which should render a strong $S_{\text {Adh }}$ based on the formation of a physical network structure. Moreover, the hydroxyl groups in virgin Glcp(1-2)-80 and Glcp-(1-4)-95 would form stable intramolecular hydrogen bonds against the mechanical stress probably at the hydrophilic domains, which may suppress the interchain binding of the hydroxyl groups. The IR absorbance wavelength of hydroxyl groups in Glcp derivatives seems to be a diagnostic tool to judge the active binding sites $(\mathrm{OH})$ to the shear-induced adhesion. It is highlighted that the benzyl groups in the

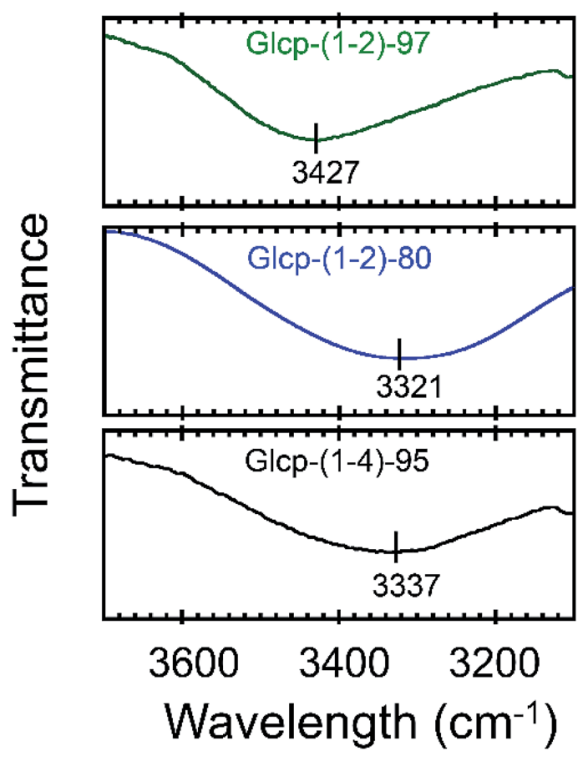

Fig. 4 Partial IR spectra of Glcp-(1-2)-97, Glcp-(1-2)-80, and Glcp-(14)-95. polymer appear to be steric protecting groups not only to kinetically stabilize the shear-induced elongated polymer structure but also to hinder the hydroxyl groups in the original coiled structure.

In conclusion, we developed a new class of adhesive materials comprising benzylated Glcp-(1-2) skeletons. We evaluated the important structural factors on the shear-induced adhesion, such as kinetically stabilized elongated structure and switchable projection of hydroxyl groups induced by stress. It was found that Glcp-(1-2)-97 exhibited optimal adhesive properties, such as higher $S_{\text {Adh }}$ than that of conventional fibrin glue and a readhesion capacity. The effects of $v$ and $G$ on $S_{\mathrm{Adh}}$ revealed that the adhesion properties of Glcp-(1-2)-97 between glass plates almost coincided with those of an ideal elastomer, implying that Glcp-(1-2)-97 had a strong energy dissipation capacity on adhesion. These systematic experiments in this study should motivate synthesis chemists to produce shearinduced adhesive materials based on the other rational combinations of polysaccharides with functional groups. As polysaccharides are predicted to be nontoxic, biodegradable, and biocompatible according to the backbone ${ }^{23-25}$ this concept of designing adhesive materials will be useful for creating polysaccharide-based stimuli-responsive bioglues in the near future.

\section{Conflicts of interest}

There are no conflicts to declare.

\section{Acknowledgements}

This work was financially supported by JSPS KAKENHI (Grant Number JP17H03070), the Hitachi Metals Materials Science Foundation, the Iketani Science and Technology Foundation, and the Grant-in-Aid for JSPS Fellows relating to JSPS Postdoctoral Fellowships for Foreign Researchers (No. P19038 for A. B. I.) from JSPS.

\section{Notes and references}

1 P. J. M. Bouten, M. Zonjee, J. Bender, S. T. K. Yauw, H. van Goor and J. C. M. van Hest, Prog. Polym. Sci., 2014, 39, 1375.

2 D. I. Tsilimigras, A. Antonopoulou, I. Ntanasis-Stathopoulos, D. Patrini, K. Papagiannopoulos, D. Lawrence and N. Panagiotopoulos, J. Thorac. Dis., 2017, 9, 568.

3 M. L. B. Palacio and B. Bhushan, Philos. Trans. R. Soc., A, 2012, 370, 2321.

4 K. Laitakari and J. Luotonen, Laryngoscope, 1989, 99, 974.

5 T. A. Springer, Blood, 2014, 124, 1412.

6 S. W. Schneider, S. Nuschele, A. Wixforth, C. Gorzelanny, A. Alexander-Katz, R. R. Netz and M. F. Schneider, Proc. Natl. Acad. Sci. U. S. A., 2007, 104, 7899.

7 A. J. Reininger, H. F. G. Heijnen, H. Schumann, H. M. Specht, W. Schramm and Z. M. Ruggeri, Blood, 2006, 107, 3537.

8 H. F. Zobel and A. M. Stephen, in Food Polysaccharides and Their Applications, ed. A. M. Stephen, G. O. Phillips and P. 
A. Williams, Taylor \& Francis, Boca Raton, FL, USA, 2006, pp. 25-85.

9 M. W. Rutenberg and D. Solarek, Starch Derivatives: Production and Uses, in Starch: Chemistry and Technology, ed. R. Whistler, J. BeMiller and E. Paschall, Elsevier, Amsterdam, 2nd edn, 1984, pp. 311-388.

10 S. O. Agboola, J. O. Akinbgala and G. B. Oguntimein, Starch/ Staerke, 1990, 42, 12.

11 A. J. Gunorubon, Research Journal in Engineering and Applied Sciences, 2012, 1, 214.

12 S. S. Shetty and Y. Koyama, Tetrahedron Lett., 2016, 57, 3657. 13 J. N. Bemiller and R. E. Wing, Carbohydr. Res., 1968, 6, 197. 14 See ESI. $\dagger$

15 A. Schallamach, Proc. Phys. Soc., London, Sect. B, 1952, 65, 657.

16 Y. B. Chernyak and A. I. Leonov, Wear, 1986, 108, 105.

17 K. Vorvolakos and M. K. Chaudhury, Langmuir, 2003, 19, 6778.
18 V. L. Popov, L. Voll, Q. Li, Y. S. Chai and M. Popov, Sci. Rep., 2014, 4(1), 3750.

19 F. Deplace, C. Carelli, S. Mariot, H. Retsos, A. Chateauminois, K. Ouzineb and C. Creton, J. Adhes., 2009, 85, 18.

20 S. S. Heddleson, D. D. Hamann and D. R. Lineback, Cereal Chem., 1993, 70, 744.

21 M. B. Novikov, A. Roos, C. Creton and M. M. Feldstein, Polymer, 2003, 44, 3561.

22 T. Kondo, Cellulose, 1997, 4, 281.

23 N. B. Shelke, R. James, C. T. Laurencin and S. G. Kumbar, Polym. Adv. Technol., 2014, 25, 448.

24 M. Rinaudo, Polym. Int., 2008, 57, 397.

25 S. Dumitriu, Polysaccharides as Biomaterials, in Polymeric Biomaterials, ed. S. Dumitriu, Taylor \& Francis, Oxfordshire, 2002, pp. 1-61. 